\title{
CRYSTALLIZATION OF AMORPHOUS RIBBON OBSERVED BY MEANS OF SCANNING TUNNELING MICROSCOPE
}

\author{
A. Witek, A. Reich, J. Rauluszkiewicz \\ Institute of Physics, Polish Academy of Sciences \\ Al. Lotników 32/46, 02-668 Warszawa, Poland \\ AND W. ZYCH \\ Institute of Physics, Warsaw Technical University \\ Koszykowa 75, 00-662 Warszawa, Poland
}

(Received November 24, 1992)

\begin{abstract}
Surfaces of amorphous ribbon $\mathrm{Fe}_{70} \mathrm{~V}_{10} \mathrm{~B}_{20}$ were observed by means of the scanning tunneling microscope before and after the annealing in vacuum. The topographic images of the air-side surfaces after annealing are similar in the $x-y$ plane and different in the $z$-direction. From the scanning tunnelling microscope images some information on the crystallization of amorphous ribbons can be deduced.
\end{abstract}

PACS numbers: $68.35 . \mathrm{Bs}, 68.55 . J k, 75.50 . \mathrm{Kj}$

\section{Introduction}

Studying of crystallization processes in metallic glasses is very important from the point of view of the phase transformations occurring in extreme conditions far from equilibrium. These processes are also interesting from the point of view of the stability of metallic glasses in potential applications. Scanning tunneling microscopy (STM) allows one to observe the surface properties of investigated species, thus it can inform on the processes of surface crystallization. On the other hand, the surface crystallization may affect some specific surface features of metallic glasses like ability to corrosion or catalytic properties. For example, magnetic anisotropy of amorphous ribbon after annealing is assumed to originate from a compressive stress induced by the formation of a crystalline surface layer with higher density [1]. Surface crystallization and its influence on magnetic properties of Fe-based metallic glass have been studied recently by Herzer and Hilzinger [2]. 
There is a number of reasons to expect that the surface crystallization in metallic glasses is preferred as compared with that of bulk. The nucleation processes and growth of crystallites can be favored by higher surface energies. The stress caused by changes of volume during crystallization can be easier relieved at the surface. Surface crystallization may be also enhanced by easier atomic migration on a free surface.

Many experimental techniques, such as X-ray diffraction, electron and optical microscopy, differential scanning calorimetry have been applied to control the crystallization processes in amorphous metals. The optical and electron microscopes suffer the specific limitation - the spatial resolution in the direction perpendicular to the surface is smaller than in the plane of surface and the surface images are not three-dimensional topographic pictures in real space.

Scanning tunneling microscopy since its discovery by Binnig and Rohrer in 1981 [3] has developed now in very attractive technique with two essential advantages: (i) atomic scale resolution and (ii) real space topography. Thus the application of STM to the observation of surfaces of amorphous metals, which due to their chemical inertness are especially suitable to the STM imaging, seems to be very attractive.

The aim of this paper is to present the application of a scanning tunneling microscope for the observation of surface crystallization of amorphous ribbons.

\section{Scanning tunneling microscope}

The STM used in these experiments was a slightly modified version of that described by Smith [4]. The modification consists in application of bimorphic membrane as the $z$-piezopositioner for the fine distance control. Its sensitivity was equal to $100 \AA / V$. The $x$ - and $y$-positioners had the sensitivities of $36 \AA / V$. All piezoelements were calibrated with a fine inductance dilatometer-based method. The tip was a tungsten wire $\phi=0.5 \mathrm{~mm}$ sharpened electrochemically.

The STM operated in the constant current mode. Stable tunneling currents were obtained at the tip voltages ranging from $0.1 \mathrm{~V}$ to $0.5 \mathrm{~V}$ with tunneling current in the range from $1 \mathrm{nA}$ to $10 \mathrm{nA}$. All measurements were performed in air at room temperature. Some thermal drifts in STM were observed, but they could be reduced by long-term thermal stabilization.

\section{Material}

The amorphous ribbons ( $40 \mu \mathrm{m}$ thick) of the alloy $\mathrm{Fe}_{70} \mathrm{~V}_{10} \mathrm{~B}_{20}$ were produced by the cold-rolling technique in the Institute of Non-Iron Metals in Gliwice. The non-crystalline nature of amorphous ribbon was verified by X-ray analysis. Also the derivative thermal magnetogravimetry-differential thermal analysis (DTMG-DTA) measurements performed on these ribbons [5] gave the Curie and crystallization temperatures characteristic of amorphous foil.

Two samples of the same alloy $\mathrm{Fe}_{70} \mathrm{~V}_{10} \mathrm{~B}_{20}$, cut from the same place of ribbon, were investigated. One of these sample was used for the STM observations directly. The second one was annealed at the temperature of $800^{\circ} \mathrm{C}$ in the vacuum for 2 hours. 


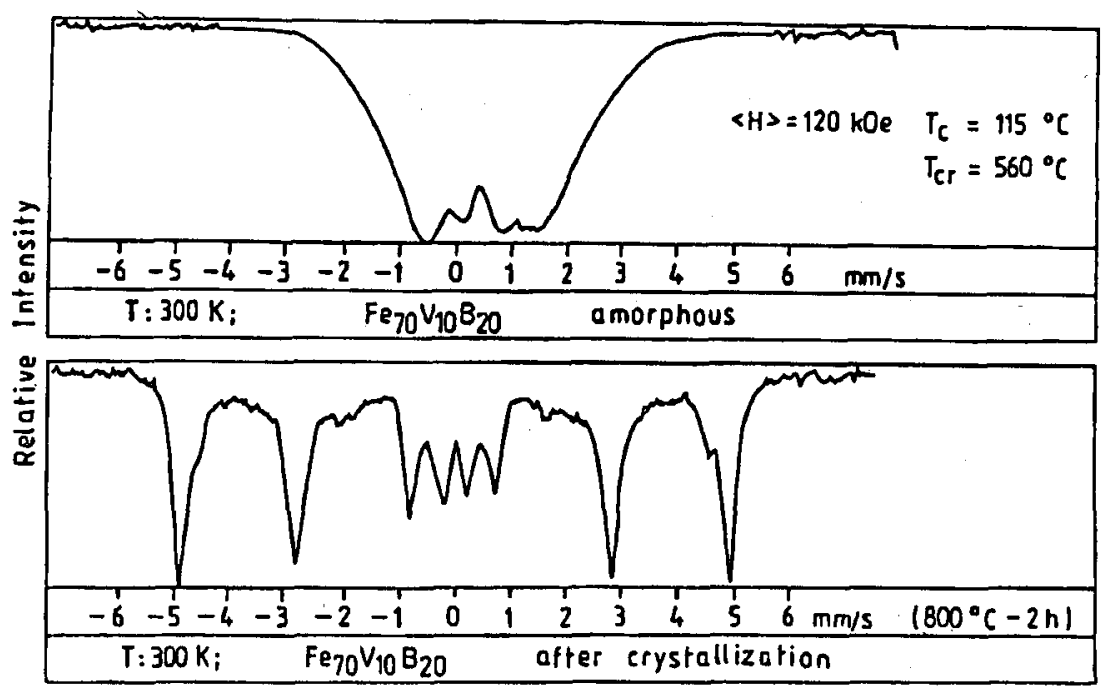

Fig. 1. The Mössbauer spectra of $\mathrm{Fe}_{70} \mathrm{~V}_{10} \mathrm{~B}_{20}$ amorphous ribbon before and after annealing.

In Fig. 1 the Mössbauer spectra of samples before and after annealing are presented. The spectrum of non-annealed sample, presented in Fig. 1a, is characteristic of amorphous alloy with overlapping Zeeman linewidths, because of low value of the hyperfine magnetic field $(120 \mathrm{kOe})$. The spectrum in Fig. $1 \mathrm{~b}$ has a component corresponding to $\alpha$-Fe structure and the supplementary doublet. The DTMG measurements give the Curie temperature typical of $\alpha$-Fe. Also the X-ray diffraction gives the $\alpha$-Fe patterns. Thus, it is no doubt that the annealing of the investigated amorphous foil for 2 hours a $800^{\circ} \mathrm{C}$ causes its crystallization.

\section{Results}

In the STM imaging of surfaces the amorphous and the annealed samples of the same alloy were used. In both cases the air sides of ribbon were studied.

The surface STM image of the first, non-annealed sample is presented in Fig. 2. The scanning area in the $x-y$ plane is $1000 \AA \times 400 \AA$, each division on both axes corresponds to $100 \AA$. The topology of surface consists of long, rather smooth hills separated by few furrows which are deep about $30 \AA$. We believe that this kind of topology of amorphous ribbon in nanometer scale is due to the finite viscosity of melt at the glassy temperature and different rates of solidification on air- and cold sides of ribbon.

The temperature of crystallization of amorphous ribbon $\mathrm{Fe}_{70} \mathrm{~V}_{10} \mathrm{~B}_{20}$ is $560^{\circ} \mathrm{C}$. After annealing at $800^{\circ} \mathrm{C}$ the sample is completely crystallized, what was confirmed by means of X-ray diffraction. The surface image of ribbon after crystallization is presented in Fig. 3. The scanning area is again $1000 \AA \times 400 \AA$ as well as divisions 


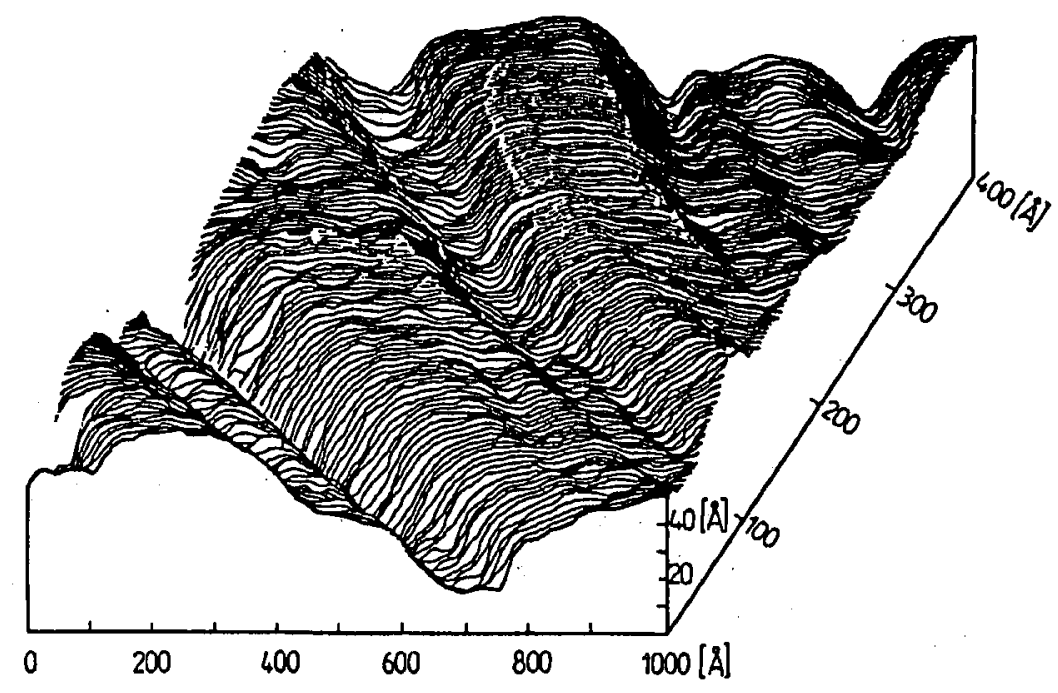

Fig. 2. STM image of the amorphous ribbon before annealing.

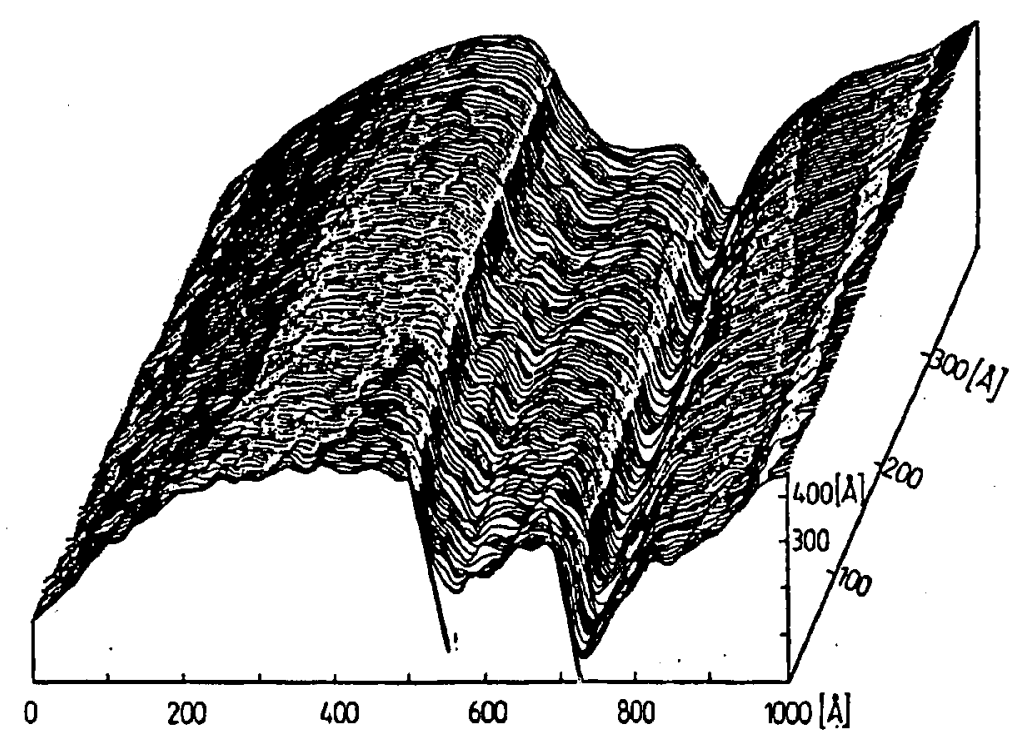

Fig. 3. STM image of the annealed ribbon. The tilted plane is subtracted.

on all axes are equal to $100 \AA$. We can see again the long hills separated by deep furrows, but in crystallized ribbons the furrows are much deeper with well-defined, flat walls. The direction of crystallized facets is indicated in Fig. 3. 


\section{Discussion}

The STM image of the $\mathrm{Fe}_{70} \mathrm{~V}_{10} \mathrm{~B}_{20}$ ribbon "as quenched" is rather smooth, with corrugations not higher than $30 \AA$. After recrystallization of the ribbon the corrugations increase considerably, the depths of furrows increase about 10 times, the walls of furrows are more flat.

These walls of a furrow seem to correspond to definite crystallographic facets created during crystallization process. The imagined crystallographic walls are sketched in Fig. 3.

The topography of both samples is very similar: long hills separated by almost parallel furrows. In the case of amorphous sample there is some similarity between the STM topography and the serpentine magnetic domain pattern which are known from the domain observations. In the case of annealed sample the topography is much more extended in the $z$-direction and the elements of crystalline facets can be recognized, which demonstrates the surface crystallization process in the ribbon. The presented above results suggest that the initial surface topology of amorphous ribbon is the matrix for further process of crystallization.

\section{Conclusion}

The surfaces of the amorphous ribbon $\mathrm{Fe}_{70} \mathrm{~V}_{10} \mathrm{~B}_{20}$ were studied by means of the scanning tunneling microscope. The air sides of the ribbons were observed before and after the crystallization caused by the annealing at $800^{\circ} \mathrm{C}$ in vacuum.

The STM images of the "as quenched" $\mathrm{Fe}_{70} \mathrm{~V}_{10} \mathrm{~B}_{20}$ ribbon are rather smooth with small corrugations of the order of $30 \AA$. After recrystallization of this ribbon the corrugations increase, the depths of furrows increase about 10 times. The walls of furrows seem to correspond to definite-crystallographic facets growing during crystallization. The topographies of ribbon before and after crystallization are similar in the $x-y$ plane, but are different in the $z$-direction.

The initial surface topography of amorphous ribbon seems to be a matrix for farther processes of crystallization.

\section{Acknowledgments}

Thanks are due to Dr. Kolano and Mr. Gawior from the Institute of Non-Iron Metals in Gliwice for the fabrication of amorphous ribbons.

\section{References}

[1] H.N. Ok, A.H. Morrish, Phys. Rev. B 23, 1835 (1981).

[2] G. Herzer, H.R. Hilzinger, Phys. Scr. 39, 639 (1989).

[3] G. Binnig, H. Rohrer, Ch. Gerber, E. Weibel, Appl. Phys. Lett. 40, 178 (1981).

[4] D.P.E. Smith, Thesis, Stanford University, 1987.

[5] R. Moskalewicz, W. Zych, Phys. Status Solidi A 97, K43 (1986). 